\title{
Hubble-induced radiative corrections and Affleck-Dine baryogenesis
}

\author{
Rouzbeh Allahverdi and Manuel Drees \\ Physik Department, TU München, James Frank Strasse, D-85748, Garching, Germany \\ Anupam Mazumdar \\ The Abdus Salam International Center for Theoretical Physics, I-34100 Trieste, Italy
}

(Received 15 October 2001; published 21 February 2002)

\begin{abstract}
We examine the viability of the Affleck-Dine mechanism for baryogenesis under radiatively induced running of soft breaking (mass) $)^{2}$ of the flat directions stemming from a nonzero energy density of the inflaton during inflation. A major difference from analogous phenomenological studies is that the horizon radius provides a natural infrared cutoff to the quantum corrections in this case. We identify different scenarios which may arise and point out that the $H_{u} L$ flat direction remains the most promising flat direction, since it is largely independent of uncertainties about high scale physics and details of the inflationary model.
\end{abstract}

DOI: $10.1103 /$ PhysRevD.65.065010

PACS number(s): 12.60.Jv, 11.30.Fs, 98.80.Cq

\section{INTRODUCTION}

The Affleck-Dine (AD) mechanism [1] provides an elegant model for generating the observed baryon asymmetry of the Universe (BAU) in the framework of supersymmetry; alternative scenarios include grand unified theory (GUT) baryogenesis [2], electroweak baryogenesis [3] and leptogenesis [4]. In this scenario some squarks and/or sleptons acquire a large vacuum expectation value (VEV) along a flat direction of the scalar potential of the minimal supersymmetric standard model (MSSM) during an inflationary epoch (for reviews, see [5]). A baryon (or lepton) number violating operator induced by new physics at a high scale and a large (spontaneously) $C$ and $C P$ violating phase, provided by the initial VEV along the flat direction, together with the out of equilibrium condition after inflation, satisfies all three requirements for the generation of baryon asymmetry [6]. The "AD field" describing the flat direction starts oscillating once its mass exceeds the Hubble expansion rate $H$. At the same time some baryon and/or lepton number violating operator produces a torque which leads to a spiral motion of the real and imaginary parts of the VEV in the complex plane. This results in a baryon (lepton) asymmetry once the comoving number density of the AD particles is frozen at sufficiently late times [1].

In the early Universe the nonzero energy density of the inflaton field is the dominant source of supersymmetry breaking. This has an important consequence in models of local supersymmetry where scalar fields generally acquire a soft supersymmetry breaking (mass) ${ }^{2}$ component (called Hubble-induced from now on) proportional to $H^{2}$ [7-9]. The effect of such a mass term crucially depends on the size and sign of the constant of proportionality. A positive (mass) ${ }^{2}$ $\ll H^{2}$ will not change the analysis of the original scenario [1]. On the other hand, for a (mass) ${ }^{2} \geqslant H^{2}$ the flat direction settles at the origin during inflation and hence cannot be used to generate BAU. It has been shown that the AD mechanism leads to interesting amounts of BAU only for a (mass) ${ }^{2}$ $<9 / 16 H^{2}[10]$. Perhaps the most interesting case occurs for a (mass) $)^{2} \sim-H^{2}$, since it naturally leads to a nonzero VEV of the flat direction before the onset of its oscillations. This can be realized at the tree level in simple extensions of minimal supergravity models $[8,9]$, and from one-loop corrections to the Kähler potential in no-scale supergravity models [11].

A detailed examination of the scenario with (mass) ${ }^{2} \sim$ $-H^{2}$, including a systematic treatment of nonrenormalizable superpotential terms which lift the flat direction, has been performed in Ref. [9]. Let us denote the AD field describing a generic direction in the scalar potential of the MSSM which is $D$ - and $F$-flat at the renormalizable superpotential level ${ }^{1}$ by $\phi$. This flat direction is lifted by a term in the superpotential of the form

$$
W \supseteq \frac{\lambda_{n} \Phi^{n}}{n M^{n-3}},
$$

where $\Phi$ is the superfield comprising $\phi$ and its fermionic partner, $M$ is the scale of new physics which induces the above term, and $\lambda_{n}$ is an $\mathcal{O}(1)$ number. Supersymmetry breaking by the inflaton energy density and by the hidden sector result in the terms

$$
\begin{gathered}
-C_{I} H^{2}|\phi|^{2}+\left(a \lambda_{n} H \frac{\phi^{n}}{n M^{n-3}}+\text { H.c. }\right)+m_{\phi, 0}^{2}|\phi|^{2} \\
+\left(A_{\phi, 0} \lambda_{n} \frac{\phi^{n}}{n M^{n-3}}+\text { H.c. }\right)
\end{gathered}
$$

in the scalar potential. The first and the third terms are the Hubble-induced and low-energy soft mass terms respectively, while the second and the fourth terms are the Hubbleinduced and low-energy $A$ terms respectively. The Hubbleinduced soft terms typically dominate the low-energy ones

\footnotetext{
${ }^{1} D$-flat directions of the MSSM are classified by gauge invariant monomials of the scalar fields of the theory, $\Pi_{i=1}^{N} \varphi_{i}$; the AD field is then defined as the linear combination $\phi \equiv\left(\sum_{i=1}^{N} \varphi_{i}\right) / \sqrt{N}$. For a detailed discussion of this, as well as the lowest-dimensional operator in the superpotential which can lift a specific flat direction, see Ref. [12].
} 
for $H>m_{0}$, where $m_{0} \sim m_{\phi, 0} \sim \mathcal{O}(\mathrm{TeV})$. If $C_{I}>0$, the absolute value of the $\mathrm{AD}$ field during inflation settles at the minimum given by

$$
|\phi| \simeq\left(\frac{C_{I}}{(n-1) \lambda_{n}} H_{I} M^{n-3}\right)^{1 / n-2},
$$

with $H_{I}$ being the Hubble constant during the inflationary epoch. ${ }^{2}$ If $|a|$ is $\mathcal{O}(1)$, the phase $\theta$ of $\langle\phi\rangle$ is related to the phase of $a$ through $n \theta+\theta_{a}=\pi$; otherwise $\theta$ will take some random value, which will generally be of $\mathcal{O}(1)$. After inflation, $\langle\phi\rangle$ initially continues to track the instantaneous local minimum of the scalar potential, which can be derived by replacing $H_{I}$ with $H(t)$ in Eq. (3). Once $H \simeq m_{0}$, the lowenergy soft terms take over. Then the (mass) ${ }^{2}$ of $\phi$ becomes positive and $\langle\phi\rangle$ moves in a nonadiabatic way, since the phase of $\langle\phi\rangle$ during inflation differs from the phase of $A$. As a result $\langle\phi\rangle$ starts a spiral motion in the complex plane, which leads to generation of a net baryon and/or lepton asymmetry [9]. Recently it has been noticed that various thermal effects from reheating can be substantial which might trigger the motion of the flat direction at an earlier time and change the yielded BAU $[13,14]$. Detailed studies of AD leptogenesis have been done which take these thermal effects into account [15].

All fields which have gauge or Yukawa couplings to the AD field contribute to the logarithmic running of its (mass) ${ }^{2}$. Therefore, one should study the evolution of the flat direction (mass) ${ }^{2}$ from some higher scale such as $M_{\mathrm{GUT}}$ down ${ }^{3}$ to low scales in order to determine the location of the true minimum of the potential and, ultimately, examine the viability of a given flat direction for the $\mathrm{AD}$ mechanism. The running of low-energy soft breaking masses has been studied in great detail in the context of MSSM phenomenology [17], in particular in connection with radiative electroweak symmetry breaking [18]. In this paper we perform similar studies in a cosmological setup for the AD mechanism.

\section{SCALE DEPENDENCE OF THE FLAT DIRECTION}

We start with a brief review of the running of the soft breaking (mass) ${ }^{2}$ of the MSSM scalars. The one-loop beta functions for the (mass) ${ }^{2}$ of the Higgs doublet $H_{u}$ which couples to the top quark, the right-handed stop $\tilde{u}_{3}$, the left-

\footnotetext{
${ }^{2}$ We have ignored the term $\propto a$ in Eq. (3). If $C_{I}>0$, the $a$ term will not change the vev qualitatively. On the other hand, even for $C_{I}$ $<0$ the potential (2) will have a minimum at a nonvanishing VEV if $|a|^{2}>4(n-1) C_{I}$. However, the origin will also be a minimum in this case. The viability of the $\mathrm{AD}$ mechanism then depends on which minimum the AD field will "choose" during inflation. Because of this complication we do not pursue the case with $C_{I}<0$ and large $|a|$ any further.

${ }^{3}$ We conservatively choose $M_{\mathrm{GUT}} \simeq 2 \times 10^{16} \mathrm{GeV}$ as the scale where SUSY breaking is transmitted to the visible sector, in order to avoid uncertainties about physics between $M_{\mathrm{GUT}}$ and $M_{\text {Planck }}$. We further notice that in M-theory scenarios the GUT scale also represents the string scale [16].
}

handed doublet of third generation squarks $\widetilde{Q}_{3}$ and the $A$ parameter $A_{t}$ associated with the top Yukawa interaction are [19]

$$
\begin{aligned}
\frac{d}{d q} m_{H_{u}}^{2}= & \frac{3 h_{t}^{2}}{8 \pi^{2}}\left(m_{H_{u}}^{2}+m_{\tilde{Q}_{3}}^{2}+m_{\tilde{u}_{3}}^{2}+\left|A_{t}\right|^{2}\right)-\frac{1}{2 \pi^{2}}\left(\frac{1}{4} g_{1}^{2}\left|m_{1}\right|^{2}\right. \\
& \left.+\frac{3}{4} g_{2}^{2}\left|m_{2}\right|^{2}\right), \\
\frac{d}{d q} m_{\tilde{u}_{3}}^{2}= & \frac{2 h_{t}^{2}}{8 \pi^{2}}\left(m_{H_{u}}^{2}+m_{\tilde{Q}_{3}}^{2}+m_{\tilde{u}_{3}}^{2}+\left|A_{t}\right|^{2}\right)-\frac{1}{2 \pi^{2}}\left(\frac{4}{9} g_{1}^{2}\left|m_{1}\right|^{2}\right. \\
& \left.+\frac{4}{3} g_{3}^{2}\left|m_{3}\right|^{2}\right), \\
\frac{d}{d q} m_{\tilde{Q}_{3}}^{2}= & \frac{h_{t}^{2}}{8 \pi^{2}}\left(m_{H_{u}}^{2}+m_{\tilde{Q}_{3}}^{2}+m_{\tilde{u}_{3}}^{2}+\left|A_{t}\right|^{2}\right)-\frac{1}{2 \pi^{2}}\left(\frac{1}{36} g_{1}^{2}\left|m_{1}\right|^{2}\right. \\
& \left.+\frac{3}{4} g_{2}^{2}\left|m_{2}\right|^{2}+\frac{4}{3} g_{3}^{2}\left|m_{3}\right|^{2}\right), \\
\frac{d}{d q} A_{t}= & \frac{3 h_{t}^{2}}{8 \pi^{2}} A_{t}-\frac{1}{2 \pi^{2}}\left(\frac{13}{36} g_{1}^{2} m_{1}+\frac{3}{4} g_{2}^{2} m_{2}+\frac{4}{3} g_{3}^{2} m_{3}\right) .
\end{aligned}
$$

Here $q$ denotes the logarithm of the scale; this could be an external energy or momentum scale, but in the case at hand the relevant scale is set by the $\operatorname{VEV}(s)$ of the fields themselves. $h_{t}$ is the top Yukawa coupling, while $g_{1} ; g_{2} ; g_{3}$ and $m_{1} ; m_{2} ; m_{3}$ are gauge couplings and soft breaking gaugino masses of the $U(1)_{Y} ; S U(2) ; S U(3)$ subgroups respectively. If $h_{t}$ is the only large Yukawa coupling (i.e., as long as $\tan \beta$ is not very large), the beta functions for the (mass) ${ }^{2}$ of squarks of the first and second generations and the sleptons only receive significant contributions from gauge or gaugino loops. A review of these effects can be found in Ref. [17]. Here we only mention the main results for universal boundary conditions, where at $M_{\mathrm{GUT}}$ the (mass) ${ }^{2}$ of all scalars is $m_{0}^{2}$ and the gauginos have the common soft breaking mass $m_{1 / 2}$. For a low value ${ }^{4}$ of $\tan \beta=1.65$,

$$
m_{H_{u}}^{2} \simeq-\frac{1}{2} m_{0}^{2}-2 m_{1 / 2}^{2}
$$

at the weak scale, while $m_{\tilde{u}_{3}}^{2}$ and $m_{\widetilde{Q}_{3}}^{2}$ remain positive. The soft breaking (mass) ${ }^{2}$ of the first and second generations of squarks is $\simeq m_{0}^{2}+(5-7) m_{1 / 2}^{2}$, while for the right-handed

\footnotetext{
${ }^{4}$ This value corresponds to the case of maximal top Yukawa coupling, so called fixed point scenario [20,21], since this maximal coupling at the weak scale is approached from a wide range of choices for $h_{t}$ at the GUT scale. Such a low value of $\tan \beta$ is excluded by Higgs boson searches at the CERN $e^{+} e^{-}$collider LEP [22], unless one allows stop masses well above $1 \mathrm{TeV}$. We nevertheless include this scenario in our discussion since it represents an extreme case.
} 
and left-handed sleptons one gets $\simeq m_{0}^{2}+0.1 m_{1 / 2}^{2}$ and $\simeq m_{0}^{2}$ $+0.5 m_{1 / 2}^{2}$, respectively. The important point is that the sum $m_{H_{u}}^{2}+m_{L}^{2}$, which describes the mass in the $H_{u} L$ flat direction, is driven to negative values at the weak scale only for $m_{1 / 2} \gtrsim m_{0}$. This is intuitively understandable since Eqs. (4) have a fixed point solution [21] $m_{H_{u}}^{2}+m_{\tilde{u}_{3}}^{2}+m_{\tilde{Q}_{3}}^{2}=A_{t}=0$ when $m_{1 / 2}=0$.

Similarly one could follow the evolution of the soft breaking terms when the Hubble-induced supersymmetry breaking is dominant, i.e., for $H>\mathcal{O}(\mathrm{TeV})$. However, some differences arise in this case. For the low-energy supersymmetry breaking case, constraints from the weak scale (e.g., realization of electroweak symmetry breaking, and experimental limits on the sparticle masses) give information about $m_{0}^{2}$ and $m_{1 / 2}$. Together with fine tuning arguments, these constraints imply that $m_{0}^{2}>0$ and $m_{0} ; m_{1 / 2}$ are $\mathcal{O}(\mathrm{TeV})$. This is different from the Hubble-induced supersymmetry breaking case, where $m_{0}^{2}$ and $m_{1 / 2}$ are determined by the scale of inflation (and the form of the Kähler potential). At low scales the Hubble-induced terms are completely negligible, because at temperature $T \sim M_{W}, H \sim \mathcal{O}(1) \mathrm{eV}$, and at present the Hubble parameter is $H_{0} \sim \mathcal{O}\left(10^{-33}\right) \mathrm{eV}$.

There exists an even more fundamental difference between the two cases. In Minkowski spacetime the contribution of a given loop to a beta function freezes at a scale of the order of the mass of the particle in the loop. In an expanding Universe the horizon radius $\propto H^{-1}$ defines an additional natural infrared cutoff for the theory. The reason is that the particle description ceases to be physically meaningful once the Compton wavelength of a particle exceeds the horizon radius. The masses of particles which are coupled to the $\mathrm{AD}$ field consist of two parts: a supersymmetry preserving part proportional to the VEV $\langle\phi\rangle$, and the Hubble-induced supersymmetry breaking part. The contribution of a given loop to a beta function should thus be frozen at a scale which is the larger of $|\langle\phi\rangle|$ and $H$ (recall that $h_{t}$ and gauge couplings are close to one). In particular, if the squared mass of the $\mathrm{AD}$ field is positive at very large scales but turns negative at some intermediate scale $Q_{c}$, the origin of the $\mathrm{AD}$ potential will cease to be a minimum provided the Hubble parameter is less than $Q_{c}$. On the other hand, if $m_{\phi}^{2}<0$ at the GUT scale, its running should already be terminated at the scale $|\langle\phi\rangle|$ determined by Eq. (3). ${ }^{5}$ In the following two subsections we therefore discuss the cases of positive and negative GUT-scale (mass) ${ }^{2}$ for the AD field separately.

\footnotetext{
${ }^{5}$ Here we note that the Hubble cutoff usually plays no role in loop corrections to the inflaton potential. In most inflationary models the masses of the fields which may run in the loop are larger than the Hubble expansion during inflation due to the presence of a finite coupling to the inflaton. This will happen if the inflaton (time varying) VEV is large and the couplings are not very small. In those cases, which are somewhat similar to our case with $C_{I}>0$, one could right away trust the usual loop calculation evaluated in a flat space time background [23].
}

\section{A. The case with $C_{I} \approx-1$}

In this case all scalar fields roll towards the origin very rapidly and settle there during inflation if radiative corrections to their (mass) ${ }^{2}$ are negligible. A typical AD field $\phi$ is a linear combination $\phi=\sum_{i=1}^{N} a_{i} \varphi_{i}$ of the MSSM scalars $\varphi_{i}$, implying that $m_{\phi}^{2}=\sum_{i=1}^{N}\left|a_{i}\right|^{2} m_{\varphi}^{2}$. As mentioned before, the running of $m_{\phi}^{2}$ crucially depends on $m_{1 / 2}$. A Hubble-induced gaugino mass can be produced from a (nonminimal) dependence of the gauge superfield kinetic terms on the inflaton field. Generally the gauge superfield kinetic terms must depend on the field(s) of the hidden or secluded sector in order to obtain gaugino masses of roughly the same order as (or larger than) scalar masses, as required by phenomenology. Having $m_{1 / 2} \sim H$ thus appears to be quite natural unless an $R$ symmetry forbids terms which are linear in the inflaton superfield [9]. The same also holds for the Hubble-induced $A$ terms. The $\mu$ term is a bit different. Since it does not break supersymmetry, there is a priori no reason to assume that $\mu$ of order $H$ will be created. However, it seems more appealing to evoke some mechanism $[24,25]$ that naturally produces $\mu$ of order of the soft breaking masses in Minkowski space. A $\mu$ term of order $H$ can probably be realized in the models of Ref. [24], but seems unlikely to emerge in those of Ref. [25]. We will therefore treat $\mu$ as a free parameter. We will see below that small values of $\mu$ are favored.

We considered sample cases with $^{6} m_{1 / 2}=H ; 3 H ; H / 3$, $A_{t}\left(M_{\mathrm{GUT}}\right)= \pm H ; \pm 3 H ; \pm H / 3, \quad h_{t}\left(M_{\mathrm{GUT}}\right)=2, \quad 0.5 \quad$ and $g_{1}\left(M_{\mathrm{GUT}}\right)=g_{2}\left(M_{\mathrm{GUT}}\right)=g_{3}\left(M_{\mathrm{GUT}}\right)=0.71$. We then followed the running of scalar soft masses from $M_{\mathrm{GUT}}$ down to $10^{3} \mathrm{GeV}$, where the low-energy supersymmetry breaking becomes dominant.

The main observation is that only the $H_{u} L$ flat direction can acquire a negative (mass) ${ }^{2}$ at low scales. In this case $m_{\phi}^{2}=\left(m_{H_{u}}^{2}+m_{L}^{2}+\mu^{2}\right) / 2$, where the last term is the contribution from the Hubble-induced $\mu$ term. The results for this case are summarized in Table $\mathrm{I}$, for $\mu\left(M_{\mathrm{GUT}}\right) \lesssim H / 4$, so that the contribution $\propto \mu^{2}$ to $m_{\phi}^{2}$ is negligible. In general $m_{\phi}^{2}$ changes sign at a higher scale for $h_{t}\left(M_{\mathrm{GUT}}\right)=2$. This is expected since a larger Yukawa coupling naturally maximizes the running of $m_{H_{u}}^{2}$. Furthermore, the difference between $A_{t} / m_{1 / 2}<0$ and $A_{t} / m_{1 / 2}>0$ becomes more apparent as $\left|A_{t} / m_{1 / 2}\right|$ increases and $h_{t}$ decreases. The quasi fixed-point value of $A_{t} / m_{1 / 2}$ is positive [21]. Positive input values of $A_{t}$ will thus lead to positive $A_{t}$ at all scales, but a negative $A_{t}\left(M_{\mathrm{GUT}}\right)$ implies that $A_{t} \simeq 0$ for some range of scales, which diminishes its effect in the RGE, see Eq. (4). The sign of $A_{t}\left(M_{\mathrm{GUT}}\right)$ is more important for smaller $h_{t}$, since then $A_{t} / m_{1 / 2}$ will evolve less rapidly.

We also notice that the squared mass of the $H_{u} L$ flat direction does not change sign when $m_{1 / 2}=H / 3$, except for ${ }^{7}$ $A_{t}= \pm 3 H$ and $h_{t}=0.5$. This can be explained by the fact that

\footnotetext{
${ }^{6}$ The renormalization group equation (RGE) (4) for $A_{t}$ shows that the relative sign between $A_{t}$ and $m_{1 / 2}$ matters since it affects the running of $\left|A_{t}\right|$ and, subsequently, scalar soft masses. Without loss of generality we take the common gaugino mass $m_{1 / 2}$ to be positive.

${ }^{7}$ For this choice of parameters, $A_{t}$ initially runs very slowly. It will therefore remain large for some time and helps $m_{H_{u}}^{2}$ to decrease quickly towards lower scales.
} 
TABLE I. The scale $Q_{c}$ (in $\mathrm{GeV}$ ) where the squared mass of the AD field describing the $H_{u} L$ flat direction changes sign, for $C_{I}=$ -1 and several values for the ratios $A_{t} / H$ and $m_{1 / 2} / H$ as well as the top Yukawa coupling $h_{t}$, all taken at scale $M_{\mathrm{GUT}}=2$ $\times 10^{16} \mathrm{GeV}$.

\begin{tabular}{lccc}
\hline \hline$A_{t} / H$ & $m_{1 / 2} / H$ & $Q_{c}\left(h_{t}=2\right)$ & $Q_{c}\left(h_{t}=0.5\right)$ \\
\hline$+1 / 3(-1 / 3)$ & $1 / 3$ & $\times$ & $\times$ \\
$+1 / 3(-1 / 3)$ & 1 & $10^{6}-10^{7}$ & $10^{3}$ \\
$+1 / 3(-1 / 3)$ & 3 & $10^{11}$ & $10^{6}-10^{7}$ \\
\hline$+1(-1)$ & $1 / 3$ & $\times$ & $\times$ \\
$+1(-1)$ & 1 & $10^{6}-10^{7}$ & $10^{5}(\times)$ \\
$+1(-1)$ & 3 & $10^{11}$ & $10^{8}\left(10^{6}\right)$ \\
\hline$+3(-3)$ & $1 / 3$ & $\times$ & $10^{7}$ \\
$+3(-3)$ & 1 & $10^{14}\left(10^{7}\right)$ & $10^{9}\left(10^{3}\right)$ \\
$+3(-3)$ & 3 & $10^{15}\left(10^{11}\right)$ & $10^{10}\left(10^{6}\right)$ \\
\hline \hline
\end{tabular}

for small $m_{1 / 2}$ and small or moderate $\left|A_{t}\right|$ we are generally close to the fixed point solution

$$
m_{H_{u}}^{2} \simeq-\frac{1}{2} H^{2}, \quad m_{\tilde{u}_{3}}^{2} \simeq 0, \quad m_{\tilde{Q}_{3}}^{2} \simeq \frac{1}{2} H^{2} .
$$

Nevertheless, even for $m_{1 / 2} \ll H$ the squared mass of the $H_{u} L$ flat direction as well as $m_{\tilde{u}_{3}}^{2}$ are $<0.2 H^{2}$ well above $1 \mathrm{TeV}$, exactly due to the fixed point solution behavior. This implies that the $H_{u} L$ flat direction can still be viable [10]. Flat directions built out of $\tilde{u}_{3}$ will be marginal at best, since the reduction of $m_{\tilde{u}_{3}}^{2}$ will be diluted by other contributions to $m_{\phi}^{2}$ that are not reduced by RG running; e.g. for the $U_{3} D_{1} D_{2}$ flat direction we find $m_{\phi}^{2}>2 H^{2} / 3$ at all scales.

The AD mechanism should always work if $Q_{c}>H_{I}$, since then the global minimum of the potential during inflation is located at $|\langle\phi\rangle| \neq 0$. Note that in this case the VEV $|\langle\phi\rangle|$ is usually determined by $Q_{c}$ rather than by Eq. (3). For scales close to $Q_{c}$ the mass term in the scalar potential Eq. (2) can be written as $\beta_{\phi} H^{2}|\phi|^{2} \log \left(|\phi| / Q_{c}\right)$, where the coefficient $\beta_{\phi}$ can be obtained from the RGE. If $\beta_{\phi}>0$, which is true for the $H_{u} L$ flat direction for $C_{I}<0$, this term will reach a minimum at $\log \left(|\phi| / Q_{c}\right)=-1$. If $Q_{c}<\left(H_{I} M_{\mathrm{GUT}}^{n-3}\right)^{1 / n-2}$ the nonrenormalizable contributions to the scalar potential are negligible for $|\phi| \sim Q_{c}$, so that the minimum of the quadratic term essentially coincides with the minimum of the complete potential given by Eq. (2). In models of high scale inflation (e.g., chaotic inflation models), the Hubble constant during inflation $H_{I}$ can be as large as $10^{13} \mathrm{GeV}$. This implies that $m_{\phi}^{2}$ for the $H_{u} L$ flat direction can only become negative during inflation if $m_{1 / 2}^{2} \gg H^{2}$, which includes the "no-scale" scenario studied in Ref. [11]. The region of parameter space safely allowing AD leptogenesis is much larger in models of intermediate and low scale inflation (e.g. some new inflation models) where $H_{I}$ is substantially smaller. In such models one can easily have $H_{I}<Q_{c}$ at least for the $H_{u} L$ flat direction, unless $m_{1 / 2}^{2} \ll H^{2}$ or $\mu^{2} \gtrsim m_{1 / 2}^{2}$.

If $Q_{c}<H_{I}, \phi$ settles at the origin during inflation and its post-inflationary dynamics will depend on the process of thermalization. If the inflaton decay products thermalize very slowly, $m_{\phi}^{2}$ is only subjected to zero-temperature radiative corrections and $\langle\phi\rangle$ can move away from the origin once $H \leqslant Q_{c}$; a necessary condition for this scenario is that inflatons do not directly decay to fields that are charged under $S U(3) \times S U(2) \times U(1)_{Y}$. If $Q_{c} \gg 1 \mathrm{TeV}, \phi$ will readily settle at the new minimum and AD leptogenesis can work. However, the situation will be completely different if inflatons directly decay to some matter fields. In such a case the plasma of inflaton decay products has a temperature $T$ $\sim\left(\Gamma_{d} H M_{\text {Planck }}^{2}\right)^{1 / 4}[26]\left(\Gamma_{d}\right.$ is the inflaton decay rate $)$. Thus fields which contribute to the running of $m_{\phi}^{2}$ are in thermal equilibrium (recall that the $\mathrm{AD}$ field is stuck at $\phi=0$ ) and their back reaction results in thermal corrections of order $+T^{2}$ to $m_{\phi}^{2}$. For generic models of inflation $T>H$, implying that thermal effects exceed radiative corrections. Therefore $\langle\phi\rangle$ remains at the origin at all times and AD leptogenesis will not work.

\section{B. The case with $C_{I} \approx+1$}

In this case all flat directions are viable if the running of $m_{\phi}^{2}$ is negligible. However, radiative corrections may change the sign (in this case to positive) at small VEV(s) possibly resulting in the entrapment of $\phi$ at the origin. We quantitatively studied the same sample cases as above.

The main results can be summarized as follows. The squared mass of the $\mathrm{AD}$ field for the $H_{u} L$ flat direction is always negative at small scales, unless $\mu^{2} \gtrsim H^{2} / 2$. However, for $m_{1 / 2}=3 H, m_{\phi}^{2}$ changes sign twice; it is positive for scales $Q$ between roughly $10^{14}$ and $10^{6} \mathrm{GeV}$, the precise values depending on $h_{t}$ and $A_{t}$. Slepton masses only receive positive contributions from electroweak gauge/gaugino loops. As a result, the squared mass of the $\mathrm{AD}$ field describing the $L L E$ flat direction remains negative down to $1 \mathrm{TeV}$, unless $m_{1 / 2}$ $>2 \mathrm{H}$; for $m_{1 / 2} \geqslant 3 \mathrm{H}, Q_{c} \geqslant 10^{9} \mathrm{GeV}$ even for this flat direction. The squared masses of all squarks (except $\tilde{u}_{3}$ ) change sign at $Q_{c}>1 \mathrm{TeV}$ unless $m_{1 / 2} \leq H / 3$; we find $Q_{c}$ $\simeq 10^{10}\left(10^{15}\right) \mathrm{GeV}$ for $m_{1 / 2} / H=1(3)$. This is due to the large positive contribution $\propto m_{3}^{2}$ to the squared squark masses at scales below $M_{\mathrm{GUT}}$. The corresponding values for the $U_{3} D_{i} D_{j}$ and $L Q D$ flat directions are usually somewhat smaller, due to the Yukawa terms in the $\beta$ function and the slower running of slepton masses, respectively; however, the listed values of $Q_{c}$ are still a fair approximation for these cases.

According to Eq. (3), the scale $|\langle\phi\rangle| \gg H$, above which the positive contribution to the scalar potential from the nonrenormalizable superpotential term in Eq. (1) dominates $-H^{2}$, now appears. If $Q_{c}>\left(H_{I} M_{\mathrm{GUT}}^{n-3}\right)^{1 / n-2}, m_{\phi}^{2}$ is positive for all $\operatorname{VEV}(\mathrm{s})$ and hence the flat direction will settle at the 
origin during inflation and remain there from then on. In such a case the flat direction is not viable for the AD mechanism. This can easily happen for flat directions involving squarks in models of low scale inflation, but is not likely for high scale inflationary models unless $m_{1 / 2} \gtrsim 3 H$. For $H_{I}<Q_{c}<\left(H_{I} M_{\mathrm{GUT}}^{n-3}\right)^{1 / n-2}$, feasible for some flat directions in both intermediate-high scale and low scale models, the potential during inflation has two minima, at $\langle\phi\rangle=0$ and at $|\langle\phi\rangle| \sim\left(H_{I} M_{\text {GUT }}^{n-3}\right)^{1 / n-2}$. Depending on the initial conditions, $\phi$ can roll down towards either of them and settle there but only the latter one will be useful for the $\mathrm{AD}$ mechanism. If $Q_{c}<H_{I}$, the $\mathrm{AD}$ field direction will settle at the value determined by Eq. (3) (the only minimum during inflation) and remain there afterwards. The appearance of another minimum at the origin after inflation, which is possible once $H<Q_{c}$, does not change the situation since these minima are separated by a barrier. Therefore in this case radiative corrections will not change the picture qualitatively; however, they will still modify the quantitative analysis, since $C_{I}$ in Eq. (3) will become scale-dependent.

In summary, for models of high-intermediate scale inflation the AD mechanism will not be disrupted unless $m_{1 / 2}$ $\geq 3 \mathrm{H}$. On the other hand, the $H_{u} L$ flat direction is the most promising one for low scale inflationary models, regardless of the value of $m_{1 / 2}$, provided only that the Hubble-induced $|\mu|$ is not too large. Similarly, if $m_{1 / 2} \geq 3 H$, AD leptogenesis along the $H_{u} L$ flat direction is the only viable option, but requires a relatively low scale $H_{I}$. However, $Q_{c}$ $\ll\left(H_{I} M_{\mathrm{GUT}}^{n-3}\right)^{1 / 2}$ and $|\langle\phi\rangle| \sim Q_{c}$ at the minimum of potential in this case. Thermal effects may therefore trigger an early oscillation of the flat direction, if inflaton directly decays to matter fields $[13,14]$.

\section{CONCLUSION}

In this paper we examined the $\mathrm{AD}$ mechanism for baryogenesis including radiative corrections to the Hubbleinduced soft breaking (mass) ${ }^{2}$ of the MSSM scalars. An important point is that in an expanding Universe the horizon radius provides a natural infrared cutoff to such corrections. Radiative corrections lead to interesting consequences whenever the Hubble-induced soft breaking parameters satisfy $m_{1 / 2} \geq m_{0}$, or $\left|A_{0}\right| \geq m_{0}$ with $m_{0}^{2}>0$; here $m_{0}, m_{1 / 2}$ and $A_{0}$ are the common soft breaking scalar and gaugino masses and common trilinear soft breaking parameter, respectively, all taken at the input scale $M_{\mathrm{GUT}}$. We found that the $H_{u} L$ flat direction remains viable for a large region of parameter space, in particular for both signs of $m_{0}^{2}$, as long as the Hubble-induced $\mu$ parameter satisfies $|\mu|^{2} \lesssim 1 / 4 \max \left\{m_{0}^{2}, m_{1 / 2}^{2}\right\}$. In contrast, flat directions involving squarks are only viable for $m_{0}^{2}<0$ and relatively small $m_{1 / 2}$, the precise upper bound depending on the Hubble parameter during inflation $H_{I}$. Purely sleptonic flat directions are intermediate between these two extreme cases.
It should be emphasized that the values of $m_{0}, m_{1 / 2}, A_{0}$ and $\mu$ used in this analysis have no bearing on present phenomenology. All these parameters are Hubble-induced, and thus contribute negligibly to the present-day sparticle spectrum. In particular, our analysis will go through even if present-day supersymmetry breaking is not due to gravity mediation, as long as physics at scales around $M_{\mathrm{GUT}}$ can be described by an effective supergravity theory in 4 dimensions. The only MSSM parameter which is of some importance for our analysis is the ratio of VEVs $\tan \beta$, which determines the Yukawa couplings of the quarks and leptons. However, we saw in Sec. II A that changing the top coupling at the GUT scale from 0.5 to 2.0 does not lead to large variations in $\mathrm{AD}$ phenomenology. If $\tan \beta \gg 1$, i.e. for large bottom and $\tau$ Yukawa couplings, the domain of viability for flat directions involving $\tilde{b}$ and/or $\tilde{\tau}$ fields will increase somewhat, in particular for $m_{0}^{2}>0$, but again we do not expect the situation to change qualitatively in this case.

Moreover, we do not find direct consequences for $Q$-ball $[27,28]$ production. Radiative corrections to the Hubbleinduced soft mass can affect the initial conditions at the onset of flat direction oscillations. On the other hand, $Q$-ball formation occurs during oscillations which start when the lowenergy supersymmetry breaking [9] or thermal effects [13] dominate the Hubble-induced supersymmetry breaking. Once the latter becomes subdominant, the standard analysis $[27,28]$ of the flatness of scalar potential and $Q$-ball formation will apply. However, there is an indirect connection, since $Q$-balls might evaporate if the inflaton decay products thermalize quickly, unless the reheat temperature is very low. We saw in Sec. II A that models with delayed thermalization might realize $\mathrm{AD}$ baryogenesis with $m_{0}^{2}>0$ even if $Q_{c}$ $<H_{I}$, as long as $Q_{c} \gg 1 \mathrm{TeV}$.

Finally, we reiterate that radiative corrections will not affect the AD mechanism qualitatively if there exists an $R$-symmetry which forbids the appearance of terms linear in the inflaton superfield (which would imply $m_{1 / 2},\left|A_{0}\right| \ll m_{0}$ ). On the other hand, in more general scenarios $Q_{c}$ depends very strongly (essentially exponentially) on $m_{1 / 2}$, and is thus very sensitive to details of physics at high scales. We have seen that $\mathrm{AD}$ leptogenesis from the $H_{u} L$ direction is quite robust and works (almost) independently of the size of $m_{1 / 2}$ and the sign of $m_{0}^{2}$, as long as the Hubble-induced $\mu$ term is not too large. Recall that this scenario also has the distinction of connecting baryogenesis with the neutrino sector parameters [15]. Our analysis provides an argument why the $H_{u} L$ direction might be preferred dynamically over the plethora of other possible flat directions.

\section{ACKNOWLEDGMENTS}

The authors thank A. Perez-Lorénzana for fruitful discussion. The work of R.A. and M.D. was supported by "Sonderforschungsbereich 375 für Astro-Teilchenphysik" der Deutschen Forschungsgemeinschaft. A.M. acknowledges the support of The Early Universe Network HPRN-CT-200000152. 
[1] I. Affleck and M. Dine, Nucl. Phys. B249, 361 (1985).

[2] S. Weinberg, Phys. Rev. Lett. 42, 850 (1979); D. Toussaint, S.B. Treiman, F. Wilczek, and A. Zee, Phys. Rev. D 19, 1036 (1979).

[3] V. Kuzmin, V. Rubakov, and M. Shaposhnikov, Phys. Lett. 155B, 36 (1985).

[4] M. Fukugita and T. Yanagida, Phys. Lett. B 174, 45 (1986).

[5] For reviews, see A.D. Linde, Particle Physics And Inflationary Cosmology (Harwood, Chur, Switzerland, 1990); K.A. Olive, Phys. Rep. 190, 307 (1990).

[6] A.D. Sakharov, JETP Lett. 5, 24 (1967).

[7] M. Dine, W. Fischler, and D. Nemechansky, Phys. Lett. 138B, 169 (1984); S. Bertolami and G.G. Ross, Phys. Lett. B 183, 163 (1987); G. Dvali, ibid. 355, 78 (1995).

[8] M. Dine, L. Randall, and S. Thomas, Phys. Rev. Lett. 75, 398 (1995).

[9] M. Dine, L. Randall, and S. Thomas, Nucl. Phys. B458, 291 (1996).

[10] J. McDonald, Phys. Lett. B 456, 118 (1999).

[11] M.K. Gaillard, H. Murayama, and K.A. Olive, Phys. Lett. B 355, 71 (1995).

[12] T. Gherghetta, C. Kolda, and S. Martin, Nucl. Phys. B468, 37 (1996).

[13] R. Allahverdi, B.A. Campbell, and J. Ellis, Nucl. Phys. B579, 355 (2000).

[14] A. Anisimov and M. Dine, Nucl. Phys. B619, 729 (2001).

[15] M. Fujii, K. Hamaguchi, and T. Yanagida, Phys. Rev. D 65, 043511 (2002). T. Asaka, M. Fujii, K. Hamaguchi, and T. Yanagida, ibid. 62, 123514 (2000); M. Fujii, K. Hamaguchi, and T. Yanagida, ibid. 63, 123513 (2001).
[16] P. Horava and E. Witten, Nucl. Phys. B460, 506 (1996).

[17] See, for example, M. Drees and S. Martin, hep-ph/9504324.

[18] L.E. Ibãnez and G.G. Ross, Phys. Lett. 110B, 215 (1982); L.E. Ibãnez, ibid. 118B, 73 (1982); J. Ellis, D.V. Nanopoulos, and K. Tamvakis, ibid. 121B, 123 (1983); L. Alvarez-Gaumè, J. Polchinski, and M.B. Wise, Nucl. Phys. B221, 495 (1983); L.E. Ibãñez and C. Lòpez, ibid. B233, 511 (1984); C. Kounnas, A.B. Lahanas, D.V. Nanopoulos, and M. Quiròs, ibid. B236, 438 (1984); G.F. Giudice and G. Ridolfi, Z. Phys. C 41, 447 (1988); M. Olechowski and S. Pokorski, Phys. Lett. B 214, 239 (1988); W. Majerotto and B. Mösslacher, Z. Phys. C 48, 273 (1990).

[19] See, for example, H.P. Nilles, Phys. Rep. 110, 1 (1984).

[20] C.T. Hill, Phys. Rev. D 24, 691 (1981); B. Pendelton and G.G. Ross, Phys. Lett. 98B, 291 (1981); E.A. Paschos, Z. Phys. C 26, 235 (1984); V. Barger, M.S. Berger, P. Ohmann, and R.J.N. Phillips, Phys. Lett. B 314, 351 (1993).

[21] M. Carena and C.E.M. Wagner, Nucl. Phys. B452, 45 (1995).

[22] The LEP Collaborations, hep-ex/0107029.

[23] See, for example, G. Dvali, Q. Shafi, and R. Schaefer, Phys. Rev. Lett. 73, 1886 (1994).

[24] G.F. Giudice and A. Masiero, Phys. Lett. B 206, 480 (1988); J.A. Casas and C. Muñoz, ibid. 306, 288 (1993).

[25] J.E. Kim and H.P. Nilles, Phys. Lett. B 263, 79 (1991); G.F. Giudice and E. Roulet, ibid. 315, 107 (1993).

[26] E.W. Kolb and M.S. Turner, The Early Universe (AddisonWesley, Redwood City, CA, 1990), p. 280.

[27] A. Kusenko and M. Shaposhnikov, Phys. Lett. B 418, 46 (1998).

[28] K. Enqvist and J. McDonald, Phys. Lett. B 425, 309 (1998). 